Original Research Paper

\title{
Features Reweighting and Similarity Coefficient Based Method for Email Spam Filtering
}

\author{
${ }^{1}$ Ahmed Osman Ali Elsiddig, ${ }^{2,4}$ Ammar Ahmed E. Elhadi and ${ }^{2,3}$ Ali Ahmed \\ ${ }^{I}$ University of Science and Technology - Khartoum, Sudan \\ ${ }^{2}$ Software Engineering, Mashreq University Khartoum North, Sudan \\ ${ }^{3}$ Faculty of Engineering, Karary University, Omdurman, Sudan \\ ${ }^{4}$ Department of Foundation, Inaya Medical College, Riyadh
}

Article history

Received: 28-02-2017

Revised: 09-08-2017

Accepted: 11-10-2017

Corresponding Author: Ammar Ahmed E. Elhadi

Software Engineering, Mashreq University - Khartoum North,

Sudan

E-mail: ammareltayeb@gmail.com

\begin{abstract}
Spam is flooding the Internet with many copies of the same message, in an attempt to force the message on people who would not otherwise choose to receive it. Anti spam by determining whether or not an incoming email is spam has become an important problem. One of the main characters or the problem of Spam filtering is its high dimension of space feature. For this reason, we need a reducing stage of dimensions. This study tried to cover this side from spam detection techniques by study the effect of re-weight of features. The works started by applying similarity coefficient in the dataset and then reweight the features in the dataset and applying similarity coefficient in the new data set. Finally make a Comparison between the result before and after re-weight and Comparison with feature selection method. The objective of this Thesis is: Study the similarity coefficient (Cosine and Dice) and Study the effects of the important feature to other features through the re-weight process. The most important results of this study are: Reweighting process did not improve the success rate of any of the two methods (Cosine and Dice). Also, Feature selection method led to improve detection in Cosine, while reweighting method not improve detection any of (Cosine or Dice).
\end{abstract}

Keywords: Spam, Spam Filtering, Feature Selection, Similarity Coefficient

\section{Introduction}

Email spam or junk mail, or unsolicited commercial email (Michael and Mattord, 2012) is process of sending not required email messages, frequently with commercial content, in large quantities to an indiscriminate set of recipients. Spam in email started to become a problem when the Internet was opened up to the general public in the mid-1990s. It grew exponentially over the following years and today composes some 80 to 85 percent of all the e-mail in the World, by a "conservative estimate". Pressure to make email spam illegal has been successful in some jurisdictions, but less so in others. The efforts taken by governing bodies, security systems and email service providers seem to be helping to reduce the onslaught of email spam (Wikipedia, 2015a).

Spam is no more garbage but risk since it recently includes virus attachments and spyware agents which make the recipients' system ruined, therefore, there is an emerging need for spam detection (Lee et al., 2010).

Many spam detection techniques based on machine learning algorithms have been proposed. As the amount of spam has been increased tremendously using bulk mailing tools, spam detection techniques should deal with it. For spam detection, parameters optimization and feature selection have been proposed to reduce processing overheads with guaranteeing high detection rates (Lee et al., 2010).

The techniques currently used by most anti-spam software are static, meaning that it is fairly easy to evade by tweaking the message a little. To do this, spammers simply examine the latest anti-spam techniques and find ways how to dodge them (GFI, 2011).

This paper is organized as follows. Section II describes the previous studies related to spam detection. Section III describes the material and methods used in this study. The results and discussion are illustrated in Section IV. Section V concludes the paper. 


\section{Related Work}

Statistical feature selection approach combined with similarity coefficients are used to improve the accuracy and detection rate for the spam detection and filtering (Abdelrahim et al., 2013). At the end, the study results based on email spam datasets show that the proposed approach enhanced the detection rate, false alarm rate and the accuracy. Study was proved that feature selection has a positive impact on the similarity methods used for spam filtering. Feature selection increased spams filter accuracy and detection rate. Also, the degree of similarity between spam to spam samples was increased.

One of the main characters or the problem of Spam filtering is its high dimension of space feature. The feature space that contains words or phrases in the documents has more than ten thousand features, which is a great preventive problem for many of the machine learning algorithms (Beiranvand et al., 2012). For this reason, we need a reducing stage of dimensions. The previous approaches have not taken into account the importance of weights of features and there are no previous studies discuss this topic. So, in this study, we tried to cover this side from spam detection techniques by study the effect of re-weight of features.

\section{Materials and Methods}

The data used in this study, created by Mark Hopkins, Erik Reeber, George Forman, Jaap Suermondt in Hewlett-Packard Labs. It was generated in June-July 1999. The dataset is available at ftp://ftp.ics.uci.edu/pub/machine-learningdatabases/ spambase/. The number of Instances in the data set are: $4601(1813$ Spam $=39.4 \%, 2788$ Non-Spam $=60.6 \%)$, while the number of Attributes are: 58 (57 continuous, 1 nominal class label) (Spambase Dataset).

The collection of spam e-mails came from the postmaster and individuals who had filed spam. While the collection of non-spam e-mails came from filed work and personal e-mails and hence the word 'mark' and the area code '430' are indicators of non-spam. These are useful when constructing a personalized spam filter. One would either have to blind such nonspam indicators or get a very wide collection of nonspam to generate a general purpose spam filter (Hastie et al., 2001).

In this study, used MATLAB software in process of experimentation and calculation results, Also, MS Excel used in order to organize Results. The experiment was implemented on ASUS laptop contains Intel core i3 with a $1.8 \mathrm{GHz}$ processor running Windows 7 and a memory of $4.00 \mathrm{~GB}$.

The works will start by applying similarity coefficient in the dataset and then re-weight the features in the dataset and applying similarity coefficient in the new data set. Finally make a Comparison between the result before and after reweight and Comparison with feature selection method.

\section{The Experiment Phases}

Figure 1 represents the steps that have been taken to achieve the comparison between Reweighting and features selection.

\section{Phase (1): Similarity}

In this phase the work started by applying the similarity coefficient (cosine and dice) on the spam database, the objective of this phase is to calculate the accuracy of the spam detection when apply the cosine and dice only.

\section{Cosine Similarity}

Measuring of similarity between two vectors of an inner product space that measures the cosine of the angle between them is called Cosine similarity. The cosine of $0^{\circ}$ is 1 and it is less than 1 for any other angle. It is thus a judgment of orientation and not magnitude: Two vectors with the same orientation have a Cosine similarity of 1 , two vectors at $90^{\circ}$ have a similarity of 0 and two vectors diametrically opposed have a similarity of -1 , independent of their magnitude. Cosine similarity is particularly used in positive space, where the outcome is neatly bounded in $[0,1]$ (Wikipedia, 2015c).

\section{Dice Coefficient}

Using statistic for comparing the similarity of two samples is defined Dice coefficient. It's mainly useful for ecological community data, As compared to Euclidean distance, Dice distance retains sensitivity in more heterogeneous data sets and gives less weight to outliers. The outcome in the range $[0,1]$ (Wikipedia, 2015c).

\section{Phase (2): Reweighting}

Contains two levels as follows:

- Level (1): In this level, the spam database was reweighed, by dividing the largest value in every feature on all other features in the same column

- Level (2): During this level, similarity coefficient (cosine and dice) was applied again, the objective of this level is to calculate the accuracy of spam detection after reweighting the spam database and apply cosine and dice

\section{Phase (3): Results}

In this phase Comparison was done between reweighting and feature selection in order to get the best method between them.

\section{Experiment Steps}

The objective of the experiment is to calculate the accuracy of the spam detection from the (spam database 
and Reweighted spam data base), by using similarity coefficient (Cosine and Dice), so in order to clarify the processes that has done during the experimental work, we will use a Mini sample of the spam database.

Step (1)

Selected Mini sample includes 10 spam message and 10 non spam messages (labeled with the letters A to $\mathrm{J}$ in the tables). Note that the main data base contains 4601 message (1813 Spam $=39.4 \%, 2788$ Non-Spam $=60.6 \%$ )

In this sample rows represents the spam, while columns represent features. Also rows form 1-10 is spam while other rows are non-spam.

Step (2)

In this step the similarity coefficient (Cosine and Dice) was apply in Mini sample As follows:

Cosine Formula (Wikipedia, 2015b):

$$
\frac{a}{\sqrt{(a+b)(a+c)}}
$$

Dice Formula (Wikipedia, 2015c):

$$
\frac{2 a}{2 a+b+c}
$$

where, $a+b+c$ is the total number of feature positions in the strings, $a$ is the number of features set in both spams, $b$ is the number of feature positions set in only one of the two spams, while $c$ is the number of feature positions set in only the other spam.

Now, through the use of MATLAB, the output was as follows:

- Based on the results described on Table $1-5$, it is clear that $60 \%$ is the best similarity rate, where it is in the case of Cosine was discovered 7 spams out of 10 spams

- Based on the results described on Table $6-10$, it is clear that $50 \%$ is the best similarity rate, where it is in the case of Dice was discovered 7 spams out of 10 spams

When comparing the Cosine and Dice it is clear that Cosine similarity (detect 7 spams with similarity $60 \%$ ) is best than Dice similarity (detect 7 spams with similarity $50 \%$ ).

Step (3)

At this stage, start the process of re-weight spam database, through a process of division on the largest value in the column (largest Feature) as follows:

Re - weight Features $=\frac{F(i j)}{L F(j)}$

where, as $F(i j)$ is feature in row $(i)$ and column $(j)$, while $L F(j)$ is largest feature in the column $(j)$.

Table 1. Minimum sample of the results after applying Cosine algorithm on the spam database

\begin{tabular}{llllllllll}
\hline $\mathrm{A}$ & $\mathrm{B}$ & $\mathrm{C}$ & $\mathrm{D}$ & $\mathrm{E}$ & $\mathrm{F}$ & $\mathrm{G}$ & $\mathrm{H}$ & $\mathrm{I}$ & $\mathrm{J}$ \\
\hline 0.612372 & 0.612372 & 0.408248 & 0.258199 & 0.258199 & 0.408248 & 0.408248 & 0.408248 & 0.471405 & 0.654654 \\
0.408248 & 0.875000 & 0.875000 & 0.632456 & 0.632456 & 0.500000 & 0.500000 & 0.500000 & 0.721688 & 0.801784 \\
0.258199 & 0.632456 & 0.790569 & 0.790569 & 0.790569 & 0.500000 & 0.500000 & 0.500000 & 0.866025 & 0.801784 \\
0.258199 & 0.632456 & 0.790569 & 1.000000 & 1.000000 & 0.632456 & 0.632456 & 0.632456 & 0.730297 & 0.507093 \\
0.408248 & 0.500000 & 0.500000 & 0.632456 & 0.632456 & 0.632456 & 0.632456 & 0.632456 & 0.730297 & 0.507093 \\
0.408248 & 0.500000 & 0.500000 & 0.632456 & 0.632456 & 0.500000 & 0.500000 & 1.000000 & 0.288675 & 0.267261 \\
0.408248 & 0.500000 & 0.500000 & 0.632456 & 0.632456 & 1.000000 & 0.500000 & 0.500000 & 0.577350 & 0.267261 \\
0.471405 & 0.721688 & 0.866025 & 0.730297 & 0.730297 & 0.288675 & 0.577350 & 0.288675 & 0.288675 & 0.267261 \\
0.654654 & 0.801784 & 0.801784 & 0.507093 & 0.507093 & 0.267261 & 0.267261 & 0.267261 & 0.771517 & 0.771517 \\
\hline
\end{tabular}

Table 2. Maximum value of the features after applying Cosine algorithm on the spam database

\begin{tabular}{llllllllll}
\hline A & B & C & D & E & F & G & H & I & J \\
\hline 0.654654 & 0.875 & 0.875 & 1 & 1 & 1 & 0.632456 & 1 & 0.866025 & 0.801784 \\
\hline
\end{tabular}

Table 3. Minimum value of the features after applying Cosine algorithm on the spam database

\begin{tabular}{llllllllll}
\hline A & B & C & D & E & F & G & H & I & J \\
\hline 0.258199 & 0.5 & 0.408248 & 0.258199 & 0.258199 & 0.267261 & 0.267261 & 0.267261 & 0.288675 & 0.267261 \\
\hline
\end{tabular}

Table 4. Average value of the features after applying Cosine algorithm on the spam database

\begin{tabular}{llllllllll}
\hline & $\mathrm{B}$ & $\mathrm{C}$ & $\mathrm{D}$ & $\mathrm{E}$ & $\mathrm{F}$ & $\mathrm{G}$ & $\mathrm{H}$ & $\mathrm{I}$ & $\mathrm{J}$ \\
\hline 0.43198 & 0.641751 & 0.670244 & 0.64622 & 0.64622 & 0.525455 & 0.501975 & 0.525455 & 0.605103 & 0.538412 \\
\hline
\end{tabular}


Ahmed Osma et al., / American Journal of Applied Sciences 2017, 14 (10): 983.993 DOI: $10.3844 /$ ajassp.2017.983.993

Table 5. Result of spam detection after applying Cosine algorithm on the spam database

\begin{tabular}{|c|c|c|c|c|c|}
\hline Similarity & $50 \%$ & $60 \%$ & $70 \%$ & $80 \%$ & $90 \%$ \\
\hline & 0 & 0 & 0 & 0 & 0 \\
\hline & 1 & 1 & 1 & 1 & 0 \\
\hline & 1 & 1 & 1 & 1 & 0 \\
\hline & 1 & 1 & 1 & 0 & 0 \\
\hline & 1 & 1 & 1 & 0 & 0 \\
\hline & 1 & 1 & 0 & 0 & 0 \\
\hline & 1 & 1 & 0 & 0 & 0 \\
\hline & 1 & 0 & 0 & 0 & 0 \\
\hline & 1 & 1 & 1 & 1 & 0 \\
\hline Detection & 8 & 7 & 5 & 3 & 0 \\
\hline
\end{tabular}

Table 6. Minimum sample of the results after applying Dice algorithm on the spam database

\begin{tabular}{|c|c|c|c|c|c|c|c|c|c|}
\hline A & $\mathrm{B}$ & $\mathrm{C}$ & $\mathrm{D}$ & $\mathrm{E}$ & $\mathrm{F}$ & $\mathrm{G}$ & $\mathrm{H}$ & I & $\mathrm{J}$ \\
\hline 0.545455 & 0.545455 & 0.363636 & 0.25 & 0.25 & 0.4 & 0.4 & 0.4 & 0.444444 & 0.6 \\
\hline 0.363636 & 0.875 & 0.875 & 0.615385 & 0.615385 & 0.4 & 0.4 & 0.4 & 0.714286 & 0.8 \\
\hline 0.25 & 0.615385 & 0.769231 & 0.769231 & 0.769231 & 0.4 & 0.4 & 0.4 & 0.857143 & 0.8 \\
\hline 0.25 & 0.615385 & 0.769231 & 1 & 1 & 0.571429 & 0.571429 & 0.571429 & 0.727273 & 0.5 \\
\hline 0.4 & 0.4 & 0.4 & 0.571429 & 0.571429 & 0.571429 & 0.571429 & 0.571429 & 0.727273 & 0.5 \\
\hline 0.4 & 0.4 & 0.4 & 0.571429 & 0.571429 & 0.5 & 0.5 & 1 & 0.25 & 0.222222 \\
\hline 0.4 & 0.4 & 0.4 & 0.571429 & 0.571429 & 1 & 0.5 & 0.5 & 0.5 & 0.222222 \\
\hline 0.444444 & 0.714286 & 0.857143 & 0.727273 & 0.727273 & 0.25 & 0.5 & 0.25 & 0.25 & 0.222222 \\
\hline 0.6 & 0.8 & 0.8 & 0.5 & 0.5 & 0.222222 & 0.222222 & 0.222222 & 0.769231 & 0.769231 \\
\hline
\end{tabular}

Table 7. Maximum value of the features after applying Dice algorithm on the spam database

\begin{tabular}{lllllllllll}
\hline $\mathrm{A}$ & $\mathrm{B}$ & $\mathrm{C}$ & $\mathrm{D}$ & $\mathrm{E}$ & $\mathrm{F}$ & $\mathrm{G}$ & $\mathrm{H}$ & $\mathrm{I}$ \\
\hline 0.6 & 0.875 & 0.875 & 1 & 1 & 1 & 0.571429 & 1 & 0.857143 & 0.8 \\
\hline
\end{tabular}

Table 8. Minimum value of the features after applying Dice algorithm on the spam database

\begin{tabular}{llllllllll}
\hline A & B & C & D & E & F & G & J & H \\
\hline 0.6 & 0.875 & 0.875 & 1 & 1 & 1 & 0.571429 & 1 & 0.857143 & 0.8 \\
\hline
\end{tabular}

Table 9. Average value of the features after applying Dice algorithm on the spam database

\begin{tabular}{llllllllll}
\hline $\mathrm{A}$ & $\mathrm{B}$ & $\mathrm{C}$ & $\mathrm{D}$ & $\mathrm{E}$ & $\mathrm{F}$ & $\mathrm{G}$ & $\mathrm{H}$ & $\mathrm{I}$ \\
\hline 0.405948 & 0.596168 & 0.626027 & 0.619575 & 0.619575 & 0.479453 & 0.451675 & 0.479453 & 0.582183 & 0.5151 \\
\hline
\end{tabular}

Table 10. Result of spam detection after applying Dice algorithm on the spam database

\begin{tabular}{|c|c|c|c|c|c|}
\hline Similarity & $50 \%$ & $60 \%$ & $70 \%$ & $80 \%$ & $90 \%$ \\
\hline & 1 & 1 & 0 & 0 & 0 \\
\hline & 0 & 0 & 0 & 0 & 0 \\
\hline & 0 & 0 & 0 & 0 & 0 \\
\hline & 1 & 1 & 1 & 0 & 0 \\
\hline & 1 & 0 & 0 & 0 & 0 \\
\hline & 1 & 0 & 0 & 0 & 0 \\
\hline & 1 & 0 & 0 & 0 & 0 \\
\hline & 1 & 0 & 0 & 0 & 0 \\
\hline & 1 & 1 & 1 & 1 & 0 \\
\hline Detection & 7 & 3 & 2 & 1 & 0 \\
\hline
\end{tabular}

Now, through the use of MATLAB, The output was as follows:

- Based on the results described on Table 11-16, it is clear that $60 \%$ is the best similarity rate, where it is in the case of Cosine was discovered 7 spams out of 10 spams

- Based on the results described on Table 17-21, it is clear that $50 \%$ is the best similarity rate, where it is in the case of Cosine was discovered 7 spams out of 10 spams 
Table 11. Re-weight: Represents a mini sample of the result after applying Re-weight equation into the spam database

\begin{tabular}{lllllllllll}
\hline & $\mathrm{A}$ & $\mathrm{B}$ & $\mathrm{C}$ & $\mathrm{D}$ & $\mathrm{E}$ & $\mathrm{F}$ & $\mathrm{G}$ & $\mathrm{H}$ & $\mathrm{I}$ & $\mathrm{J}$ \\
\hline 1 & 0.000000 & 1.000000 & 0.831169 & 0 & 0.156863 & 0.000000 & 0.000000 & 0.000000 & 0.000000 & 0.000000 \\
2 & 1.000000 & 0.437500 & 0.649351 & 0 & 0.068627 & 0.875000 & 0.552632 & 0.037234 & 0.000000 & 1.000000 \\
3 & 0.285714 & 0.000000 & 0.922078 & 0 & 0.602941 & 0.593750 & 0.500000 & 0.063830 & 0.695652 & 0.265957 \\
4 & 0.000000 & 0.000000 & 0.000000 & 0 & 0.308824 & 0.000000 & 0.815789 & 0.335106 & 0.336957 & 0.670213 \\
5 & 0.000000 & 0.000000 & 0.000000 & 0 & 0.308824 & 0.000000 & 0.815789 & 0.335106 & 0.336957 & 0.670213 \\
6 & 0.000000 & 0.000000 & 0.000000 & 0 & 0.906863 & 0.000000 & 0.000000 & 0.984043 & 0.000000 & 0.000000 \\
7 & 0.000000 & 0.000000 & 0.000000 & 0 & 0.941176 & 0.000000 & 0.000000 & 0.000000 & 0.000000 & 0.680851 \\
8 & 0.000000 & 0.000000 & 0.000000 & 0 & 0.921569 & 0.000000 & 0.000000 & 1.000000 & 0.000000 & 0.000000 \\
9 & 0.714286 & 0.000000 & 0.597403 & 0 & 0.29902 & 0.000000 & 0.789474 & 0.000000 & 1.000000 & 0.808511 \\
10 & 0.285714 & 0.187500 & 1.000000 & 0 & 0.093137 & 1.000000 & 1.000000 & 0.000000 & 0.065217 & 0.000000 \\
11 & 0.000000 & 0.000000 & 0.000000 & 0 & 0.000000 & 0.000000 & 0.000000 & 0.000000 & 0.000000 & 0.000000 \\
12 & 0.000000 & 0.000000 & 0.000000 & 0 & 0.000000 & 0.000000 & 0.000000 & 0.000000 & 0.000000 & 0.904255 \\
13 & 0.000000 & 0.000000 & 0.000000 & 0 & 0.000000 & 0.000000 & 0.000000 & 0.000000 & 0.000000 & 0.000000 \\
14 & 0.000000 & 0.000000 & 0.000000 & 0 & 0.000000 & 0.000000 & 0.000000 & 0.000000 & 0.000000 & 0.000000 \\
15 & 0.000000 & 0.000000 & 0.000000 & 0 & 0.000000 & 0.000000 & 0.000000 & 0.000000 & 0.000000 & 0.000000 \\
16 & 0.000000 & 0.000000 & 0.000000 & 0 & 1.000000 & 0.000000 & 0.000000 & 0.000000 & 0.000000 & 0.000000 \\
17 & 0.000000 & 0.000000 & 0.000000 & 0 & 0.000000 & 0.000000 & 0.000000 & 0.000000 & 0.000000 & 0.000000 \\
18 & 0.000000 & 0.000000 & 0.000000 & 0 & 0.000000 & 0.000000 & 0.000000 & 0.000000 & 0.000000 & 0.000000 \\
19 & 0.000000 & 0.000000 & 0.000000 & 0 & 0.000000 & 0.000000 & 0.000000 & 0.000000 & 0.000000 & 0.000000 \\
20 & 0.000000 & 0.000000 & 0.000000 & 0 & 0.000000 & 0.000000 & 0.000000 & 0.000000 & 0.000000 & 0.000000 \\
\hline
\end{tabular}

Table 12. Minimum sample of the results after applying Cosine algorithm on the Re-weight spam database

\begin{tabular}{llllllllll}
\hline $\mathrm{A}$ & $\mathrm{B}$ & $\mathrm{C}$ & $\mathrm{D}$ & $\mathrm{E}$ & $\mathrm{F}$ & $\mathrm{G}$ & $\mathrm{H}$ & $\mathrm{I}$ & $\mathrm{J}$ \\
\hline 0.612372 & 0.612372 & 0.408248 & 0.258199 & 0.258199 & 0.408248 & 0.408248 & 0.408248 & 0.471405 & 0.654654 \\
0.408248 & 0.875000 & 0.875 & 0.632456 & 0.632456 & 0.500000 & 0.500000 & 0.500000 & 0.721688 & 0.801784 \\
0.258199 & 0.632456 & 0.790569 & 0.790569 & 0.790569 & 0.500000 & 0.500000 & 0.500000 & 0.866025 & 0.801784 \\
0.258199 & 0.632456 & 0.790569 & 1.000000 & 1.000000 & 0.632456 & 0.632456 & 0.632456 & 0.730297 & 0.507093 \\
0.408248 & 0.500000 & 0.500000 & 0.632456 & 0.632456 & 0.632456 & 0.632456 & 0.632456 & 0.730297 & 0.507093 \\
0.408248 & 0.500000 & 0.500000 & 0.632456 & 0.632456 & 0.500000 & 0.500000 & 1.000000 & 0.288675 & 0.267261 \\
0.408248 & 0.500000 & 0.500000 & 0.632456 & 0.632456 & 1.000000 & 0.500000 & 0.500000 & 0.577350 & 0.267261 \\
0.471405 & 0.721688 & 0.866025 & 0.730297 & 0.730297 & 0.288675 & 0.57735 & 0.288675 & 0.288675 & 0.267261 \\
0.654654 & 0.801784 & 0.801784 & 0.507093 & 0.507093 & 0.267261 & 0.267261 & 0.267261 & 0.771517 & 0.771517 \\
\hline
\end{tabular}

Table 13. Maximum value of the features after applying Cosine algorithm on the Re-weight spam database

\begin{tabular}{llllllllll}
\hline A & B & C & D & E & F & G & H & I & J \\
\hline 0.654654 & 0.875 & 0.875 & 1 & 1 & 1 & 0.632456 & 1 & 0.866025 & 0.801784 \\
\hline
\end{tabular}

Table 14. Minimum value of the features after applying Cosine algorithm on the Re-weight spam database

\begin{tabular}{llllllllll}
\hline A & B & C & D & E & F & G & H & I & J \\
\hline 0.258199 & 0.5 & 0.408248 & 0.258199 & 0.258199 & 0.267261 & 0.267261 & 0.267261 & 0.288675 & 0.267261 \\
\hline
\end{tabular}

Table 15. Average value of the features after applying Cosine algorithm on the Re-weight spam database

\begin{tabular}{llllllllll}
\hline $\mathrm{A}$ & $\mathrm{B}$ & $\mathrm{C}$ & $\mathrm{D}$ & $\mathrm{E}$ & $\mathrm{F}$ & $\mathrm{G}$ & $\mathrm{H}$ & $\mathrm{I}$ & $\mathrm{J}$ \\
\hline 0.43198 & 0.641751 & 0.670244 & 0.64622 & 0.64622 & 0.525455 & 0.501975 & 0.525455 & 0.605103 & 0.538412 \\
\hline
\end{tabular}

Table 16. Result of spam detection after applying Cosine algorithm on the Re-weight spam database

\begin{tabular}{llllll}
\hline Similarity & $50 \%$ & $60 \%$ & $70 \%$ & $80 \%$ & $90 \%$ \\
\hline & 0 & 0 & 0 & 0 & 1 \\
\\
1 & 1 & 1 & 1 & 1 & 0 \\
& 1 & 1 & 1 & 0 & 0 \\
& 1 & 1 & 1 & 0 & 0 \\
& 1 & 1 & 0 & 0 & 0 \\
Detection & 1 & 1 & 0 & 0 & 0 \\
& 1 & 0 & 1 & 0 & 0 \\
\end{tabular}


Table 17. Minimum sample of the results after applying Dice algorithm on the Re-weight spam database

\begin{tabular}{llllllllll}
\hline $\mathrm{A}$ & $\mathrm{B}$ & $\mathrm{C}$ & $\mathrm{D}$ & $\mathrm{E}$ & $\mathrm{F}$ & $\mathrm{G}$ & $\mathrm{H}$ & $\mathrm{I}$ & $\mathrm{J}$ \\
\hline 0.545455 & 0.545455 & 0.363636 & 0.250000 & 0.250000 & 0.400000 & 0.400000 & 0.400000 & 0.444444 & 0.600000 \\
0.363636 & 0.875000 & 0.875000 & 0.615385 & 0.615385 & 0.400000 & 0.400000 & 0.400000 & 0.714286 & 0.800000 \\
0.250000 & 0.615385 & 0.769231 & 0.769231 & 0.769231 & 0.400000 & 0.400000 & 0.400000 & 0.857143 & 0.800000 \\
0.250000 & 0.615385 & 0.769231 & 1.000000 & 1.000000 & 0.571429 & 0.571429 & 0.571429 & 0.727273 & 0.500000 \\
0.400000 & 0.400000 & 0.400000 & 0.571429 & 0.571429 & 0.571429 & 0.571429 & 0.571429 & 0.727273 & 0.500000 \\
0.400000 & 0.400000 & 0.400000 & 0.571429 & 0.571429 & 0.500000 & 0.500000 & 1.000000 & 0.250000 & 0.222222 \\
0.400000 & 0.400000 & 0.400000 & 0.571429 & 0.571429 & 1.000000 & 0.500000 & 0.500000 & 0.500000 & 0.222222 \\
0.444444 & 0.714286 & 0.857143 & 0.727273 & 0.727273 & 0.250000 & 0.500000 & 0.250000 & 0.250000 & 0.222222 \\
0.600000 & 0.800000 & 0.800000 & 0.500000 & 0.500000 & 0.222222 & 0.222222 & 0.222222 & 0.769231 & 0.769231 \\
\hline
\end{tabular}

Table 18. Maximum value of the features after applying Dice algorithm on the Re-weight spam database

\begin{tabular}{llllllllll}
\hline A & B & C & D & E & F & G & H & I & J \\
\hline 0.6 & 0.875 & 0.875 & 1 & 1 & 1 & 0.571429 & 1 & 0.857143 & 0.8 \\
\hline
\end{tabular}

Table 19. Minimum value of the features after applying Dice algorithm on the Re-weight spam database

\begin{tabular}{llllllllll}
\hline $\mathrm{A}$ & $\mathrm{B}$ & $\mathrm{C}$ & $\mathrm{D}$ & $\mathrm{E}$ & $\mathrm{F}$ & $\mathrm{G}$ & $\mathrm{H}$ & $\mathrm{I}$ & $\mathrm{J}$ \\
\hline 0.25 & 0.4 & 0.363636 & 0.25 & 0.25 & 0.222222 & 0.222222 & 0.222222 & 0.25 & 0.222222 \\
\hline
\end{tabular}

Table 20. Average value of the features after applying Dice algorithm on the Re-weight spam database

\begin{tabular}{llllllllll}
\hline $\mathrm{A}$ & $\mathrm{B}$ & $\mathrm{C}$ & $\mathrm{D}$ & $\mathrm{E}$ & $\mathrm{F}$ & $\mathrm{G}$ & $\mathrm{H}$ & $\mathrm{I}$ & $\mathrm{J}$ \\
\hline 0.626027 & 0.619575 & 0.619575 & 0.479453 & 0.451675 & 0.479453 & 0.582183 & 0.5151 & 0.626027 & 0.619575 \\
\hline
\end{tabular}

Table 21 Result of spam detection after applying Dice algorithm on the Re-weight spam database

\begin{tabular}{llllll}
\hline Similarity & $50 \%$ & $60 \%$ & $70 \%$ & $80 \%$ & $90 \%$ \\
\hline & 1 & 1 & 0 & 0 & 0 \\
\\
0 & 0 & 0 & 0 & 0 & 0 \\
& 1 & 1 & 0 & 0 & 0 \\
1 & 0 & 1 & 0 & 0 \\
& 1 & 0 & 0 & 0 & 0 \\
Detection & 1 & 0 & 0 & 0 & 0 \\
\\
\hline
\end{tabular}

When comparing the Cosine and Dice after Reweight it is clear that Cosine similarity (detect 7 spams with similarity $60 \%$ ) is best than Dice similarity (detect 7 spams with similarity 50\%).

\section{Results and Discussion}

After implementation the experiment on the mini sample and from the results, it is clear that Reweight operation does not have any positive impact on the detection process.

\section{Phase (1)}

\section{Cosine and Dice Similarity}

In this phase the experiment started by calculate the value of cosine and Dice, As shown in Table 22 and 23 Best ratio of similarity is at $60 \%$, at this ratio Cosine method succeeded in identifying 1793 spam and Failure to identify 20 spam, while Dice method at the same ratio (60\%) succeeded in identifying 1796 spam and Failure to identify 17 Spam. As noted earlier, the original data base containing 1813 spam.

\section{Results}

At this phase Experiment proved that Dice similarity is best than Cosine similarity, As shown in Table 23, Dice fail in identify 17 spam while Cosine Fail in identify 20 spam.

\section{Phase (2)}

\section{Reweighting Spam Database}

During this phase reweighting operation was apply and then applied Cosine and Dice methods in the new database.

\section{Cosine and Dice Similarity after Reweighting}

As shown in Table 24 and 25, Best ratio of similarity is at $60 \%$, at this ratio Cosine method succeeded in identifying 1790 spam and Failure to 
identify 23 Spam, while Dice method at the same ratio $(60 \%)$ succeeded in identifying 1792 Spam and Failure to identify 21 Spam.

\section{Results after Reweighting}

As shown in Tables 22 to 25, Experiment proved that Dice similarity is best than Cosine similarity, Dice fail in identify 21 spam while Cosine Fail in identify 23 spam.

\section{Results of the Experiment}

From the results before and after applying reweighting clear to us the following:

- Dice similarity Give the best results in both cases (before and after reweighting)

- Reweighting process did not improve the success rate of any of the two methods (Cosine and Dice)

Figure 2. Shows the comparison between Cosine and Dice after applying features selection similarity, while Fig. 3. Shows the comparison between Cosine and Dice after applying Re-weight Process. The results of Reweighting shown in Fig. 4 and Fig. 6. clarify the comparison between Cosine and Dice before and after applying feature selection. Figure 7 shows the results obtained by (Abdelrahim et al., 2013) in their study after using similarity algorithms to detect spam.

\section{Phase (3)}

\section{Reweighting VS Feature Selection}

From the results obtained in Tables (22-25), it is clear that the process of re-weight did not have a positive impact in improving the spams detection Fig 5.

\section{Results after Features Selection}

The results from the study (Abdelrahim et al., 2013) Table 26 were as follows:

- $\quad$ The paper proved that features selection process had a positive effect in improving the accuracy of the spams detection

- Features selection process led to the improvement of detection in the Cosine, while it has a negative effect in the case of Dice

Based on the experiment and the results from the study (Abdelrahim et al., 2013), following results were Obtained Feature selection method is best than Reweighting:

- Feature selection method led to improve detection in Cosine, while reweighting method not improve detection any of (Cosine or Dice)

Table 22. Cosine and dice similarity

\begin{tabular}{llllll}
\hline Similarity & $50 \%$ & $60 \%$ & $70 \%$ & $80 \%$ & $90 \%$ \\
\hline Cosine & 1800 & 1793 & 1719 & 1155 & 239 \\
Dice & 1803 & 1796 & 1705 & 1130 & 238 \\
\hline
\end{tabular}

Table 23. Results of cosine and dice similarity

\begin{tabular}{lllll}
\hline Similarity method & Max similarity & Min similarity & Success & Fail \\
\hline Cosine & 1 & 0.19 & 1793 & 20 \\
Dice & 1 & 0.17 & 1796 & 17 \\
\hline
\end{tabular}

Table 24. Cosine and dice after reweighting

\begin{tabular}{llllll}
\hline Similarity & $50 \%$ & $60 \%$ & $70 \%$ & $80 \%$ & $90 \%$ \\
\hline Cosine & 1797 & 1790 & 1716 & 1154 & 239 \\
Dice & 1799 & 1792 & 1701 & 1129 & 238 \\
\hline
\end{tabular}

Table 25. Results of cosine and dice after reweighting

\begin{tabular}{lllll}
\hline Similarity method & Max similarity & Min similarity & Success & Fail \\
\hline Cosine & 1 & 0.19 & 1790 & 23 \\
Dice & 1 & 0.17 & 1792 & 21 \\
\hline
\end{tabular}

Table 26. Represents the results obtained by (Abdelrahim et al., 2013) in their paper after applying spam detection algorithms in the same spam database

\begin{tabular}{lllll}
\hline Similarity method & Max similarity & Min similarity & Success & Fail \\
\hline Cosine & 1 & 0.47 & 1812 & 1 \\
Dice & 1 & 0 & 1800 & 13 \\
\hline
\end{tabular}


Phase (1): Similarity

Spam detection using similarity.

\section{Phase (2):Reweighting}

Spam database Reweighting.

Spam detection using similarity after Reweighting.

Phase (3):Results

Reweighting VS features selection

Fig. 1. Experiment phases

$\mathbf{v}$ Cosine Dice

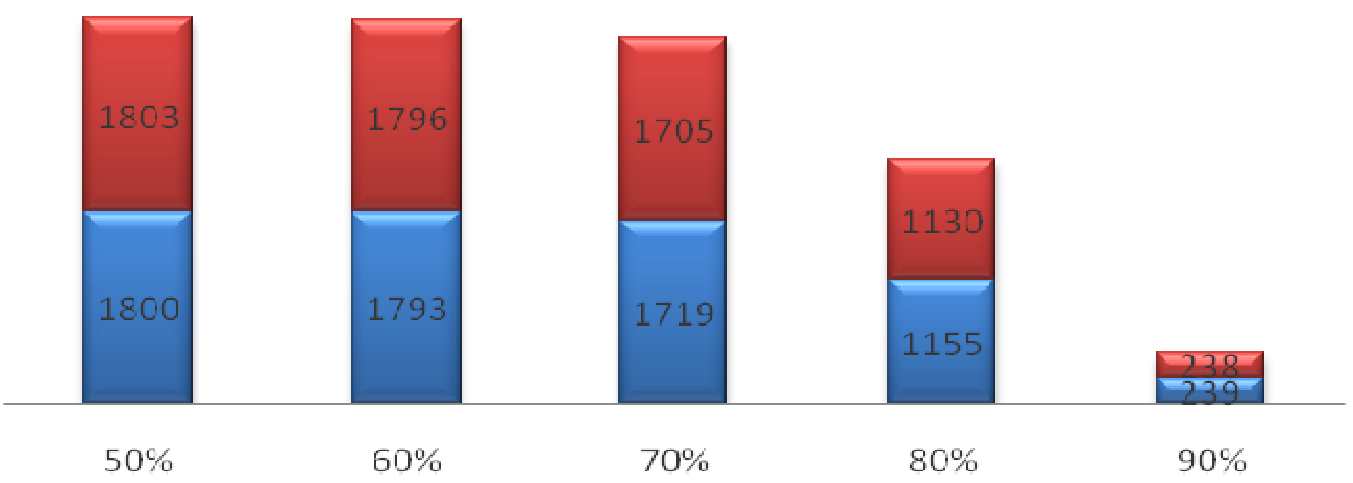

Fig. 2. Cosine and dice similarity

$\square$ Cosine $\square$ Dice

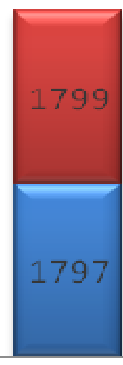

$50 \%$

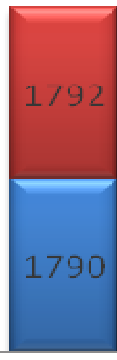

$60 \%$

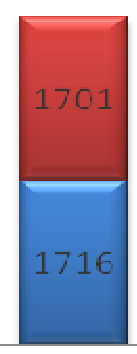

$70 \%$

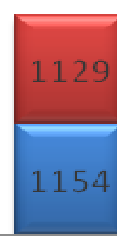

$80 \%$

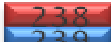

$90 \%$

Fig. 3. Cosine and dice similarity after reweighting 
$\square$ Cosine $\mathbf{D i c e}$
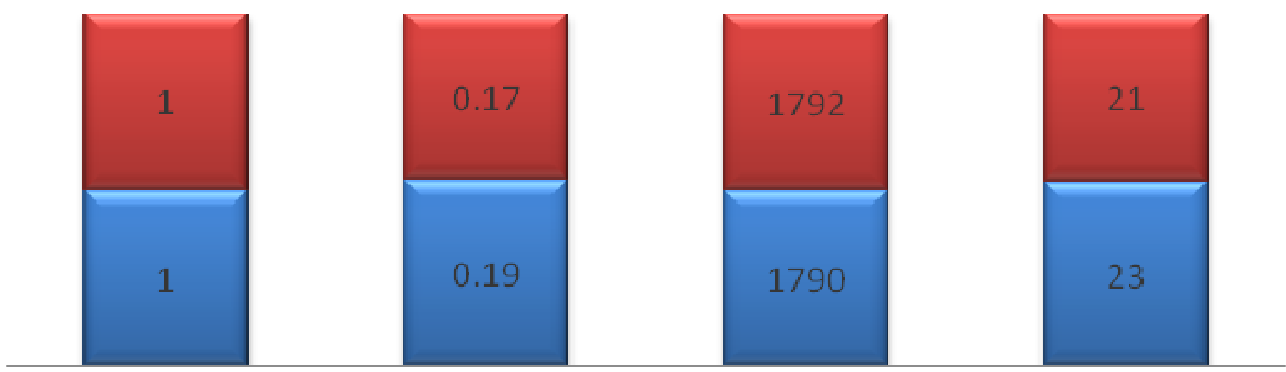

Max similarity

Minsimilarity

Success

Fail

Fig. 4. Results of cosine and dice after reweighting

\section{Cosine and Dice befor and after Reweghing}

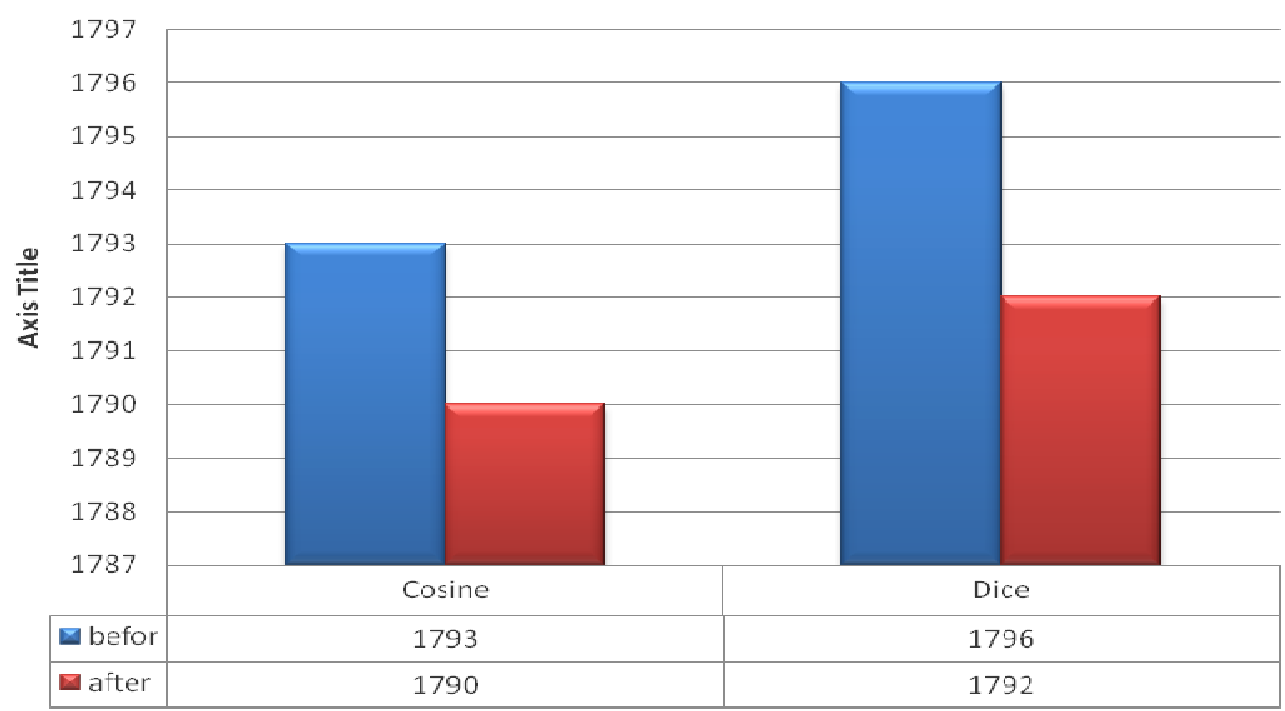

Fig. 5. Results of cosine and dice after reweighting

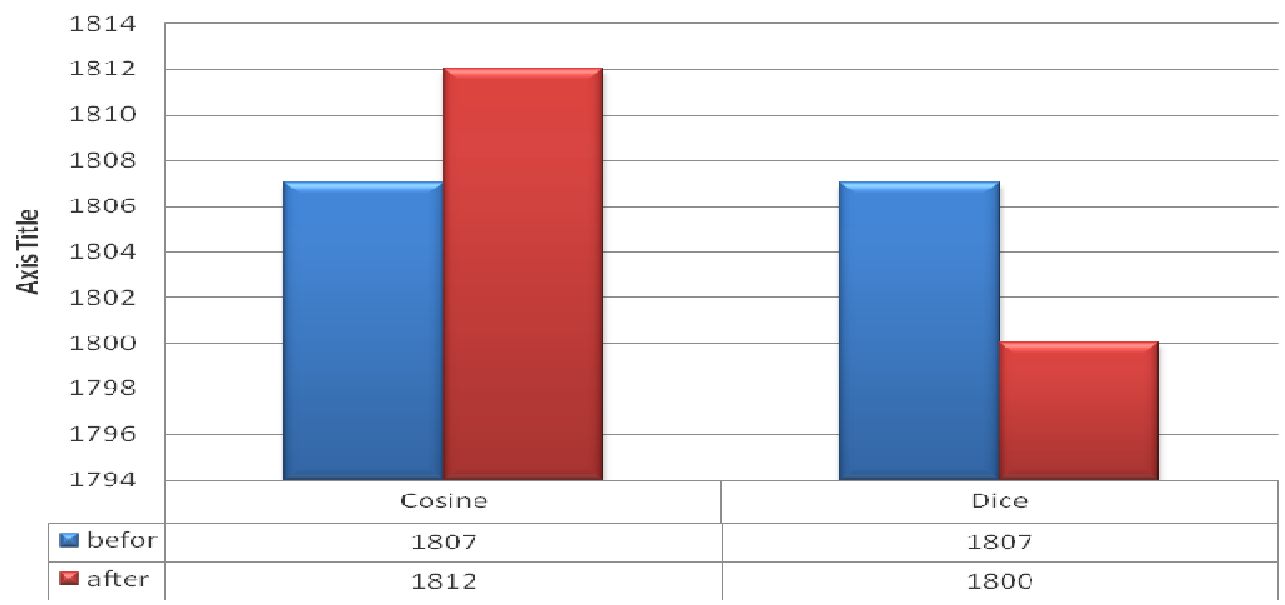

Fig. 6. Cosine and dice before and after feature selection 


\section{Results after Features Selection}

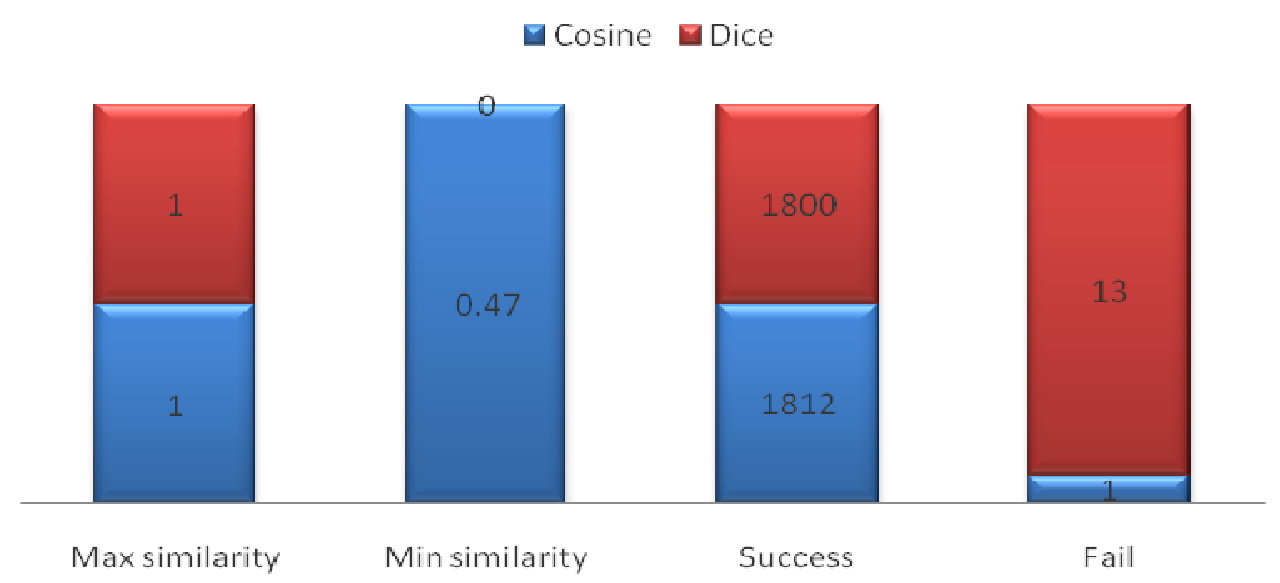

Fig. 7. Results of cosine and dice after feature selection

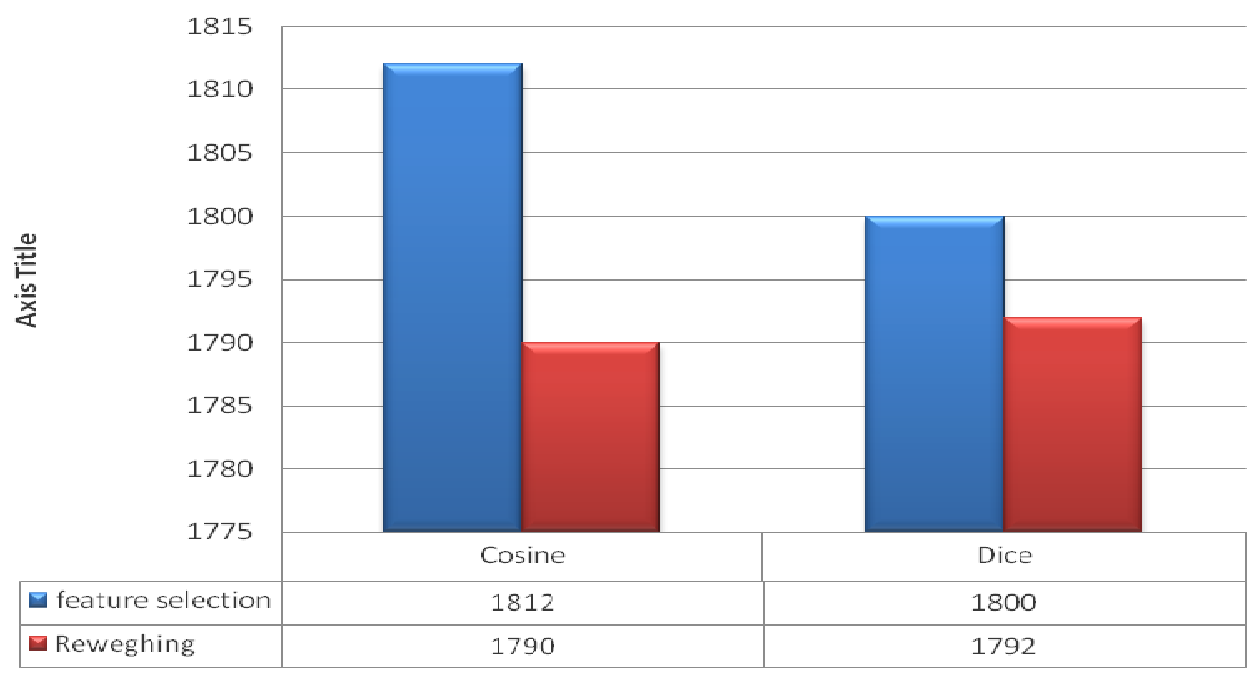

Fig. 8. Feature selection VS reweighting

\section{Recommendations and Future Research}

Based on the findings of this study, the following recommendations are offered for consideration

- Despite the findings in this research, we cannot say that the re-weight process does not have a positive impact in all cases, so it's recommended to looking for other ways to improve the process of re-weight. Because the logic indicates that there are some features have higher weight and its presence indicates a large margin that the message is spam

- Because this study did not get a positive effect after applying Cosine and Dice similarity, (Fig. 8) the study recommended re-applying this experience to other similarity coefficients

\section{Conclusion}

The result, which reached in this study is that the re-weight process did not have a positive impact on the Spam detection process, this result can be explained as follows: There are other algorithms for the process of re-weight can be tested in the future, also previous studies have shown that features selection had a positive impact in improving Spam detection, so can Combine the process of features selection with re-weight to get the best results.

\section{Acknowledgement}

The authors would like to thank our colleagues and staff at University of Science and Technology and Mashreq University for their contribution and comments. 


\section{Author's Contributions}

Ahmed Osman Ali Elsiddig: Has given a significant contribution in the preparation of this article. Produced the initial draft of the article, develop and carry out this manuscript.

Ammar Ahmed E. Elhadi and Ali Ahmed: Are the supervisors, who oversaw the overall research article, reviewed and finalized the draft of the article before it is being submitted.

\section{Ethics}

This article is original and contains unpublished material. The corresponding author confirms that all of the other authors have read and approved the manuscript and no ethical issues involved.

\section{References}

Abdelrahim, A.A.A., A. Ahmed, E. Elhadi, H. Ibrahim and N. Elmisbah et al., 2013. Feature selection and similarity coefficient based method for email spam filtering. Proceedings of the International Conference on Computing, Electrical and Electronics Engineering, Aug. 26-28, IEEE Xplore Press, Khartoum, Sudan, pp: 26-28. DOI: 10.1109/ICCEEE.2013.6634013
Beiranvand, A., A. Osareh and B. Shadgar, 2012. Spam filtering by using a compound method of feature selection. J. Acad. Applied Studies, 2: 25-31.

GFI, 2011. Why Bayesian filtering is the most effective anti-spam technology. Software.

Hastie, T., R. Tibshirani and J.H. Friedman, 2001. The Elements of Statistical Learning. 1st End., Springer, Science and Business Media.

Lee, S.M., D.S. Kim, J.H. Kim and J.S. Park, 2010. Spam detection using feature selection and parameters optimization. Proceedings of the International Conference on Complex, Intelligent and Software Intensive Systems, Feb. 15-18, IEEE Xplore Press, Krakow, Poland, pp: 15-18. DOI: $10.1109 /$ CISIS.2010.116

Michael, E.W. and H.J. Mattord, 2012. Principles of Information Security. 4th Ed, Cengage Learning, USA.

Spambase Dataset, http://ftp.ics.uci.edu/pub/machinelearning-databases/spambase/

Wikipedia, 2015a. Cosine Similarity.

Wikipedia, 2015b. Dice Coefficient.

Wikipedia, 2015c. Spamming. /Spamming 